\title{
BMJ Open Double-blind cross-over pilot trial protocol to evaluate the safety and preliminary efficacy of long-term adaptive deep brain stimulation in patients with Parkinson's disease
}

Sara Marceglia (D) ${ }^{1,2}$ Costanza Conti, ${ }^{3}$ Oleg Svanidze, ${ }^{3}$ Guglielmo Foffani, ${ }^{4,5}$ Andres M Lozano, ${ }^{6,7}$ Elena Moro, ${ }^{8}$ Jens Volkmann, ${ }^{9}$ Mattia Arlotti, ${ }^{3}$ Lorenzo Rossi, ${ }^{3}$ Alberto Priori ${ }^{10,11}$

To cite: Marceglia S, Conti C, Svanidze 0, et al. Doubleblind cross-over pilot trial protocol to evaluate the safety and preliminary efficacy of long-term adaptive deep brain stimulation in patients with Parkinson's disease. BMJ Open 2022;12:e049955. doi:10.1136/ bmjopen-2021-049955

- Prepublication history and additional supplemental material for this paper are available online. To view these files, please visit the journal online (http://dx.doi.org/10.1136/ bmjopen-2021-049955).

SM and CC contributed equally.

Received 15 February 2021 Accepted 13 December 2021

Check for updates

(C) Author(s) (or their employer(s)) 2022. Re-use permitted under CC BY-NC. No commercial re-use. See rights and permissions. Published by BMJ.

For numbered affiliations see end of article.

Correspondence to Dr Sara Marceglia; smarceglia@units.it

\section{ABSTRACT}

Introduction After several years of brain-sensing technology development and proof-of-concept studies, adaptive deep brain stimulation (aDBS) is ready to better treat Parkinson's disease (PD) using aDBS-capable implantable pulse generators (IPGs). New aDBS devices are capable of continuous sensing of neuronal activity from the subthalamic nucleus (STN) and contemporaneous stimulation automatically adapted to match the patient's clinical state estimated from the analysis of STN activity using proprietary algorithms. Specific studies are necessary to assess superiority of aDBS vs conventional DBS (CDBS) therapy. This protocol describes an original innovative multicentre international study aimed to assess safety and efficacy of aDBS vs cDBS using a new generation of DBS IPG in PD (AlphaDBS system by Newronika SpA, Milan, Italy).

Methods The study involves six investigational sites (in Italy, Poland and The Netherlands). The primary objective will be to evaluate the safety and tolerability of the AlphaDBS System, when used in cDBS and aDBS mode. Secondary objective will be to evaluate the potential efficacy of aDBS. After eligibility screening, 15 patients with PD already implanted with DBS systems and in need of battery replacement will be randomised to enter a twophase protocol, including a 'short-term follow-up' (2 days experimental sessions during hospitalisation, 1 day per each mode) and a 'long-term follow-up' (1 month at home, 15 days per each mode).

Ethics and dissemination The trial was approved as premarket study by the Italian, Polish, and Dutch Competent Authorities: Bioethics Committee at National Oncology Institute of Maria Skłodowska-Curie-National Research Institute in Warsaw; Comitato Etico Milano Area 2; Comitato Etico IRCCS Istituto Neurologico C. Besta; Comitato Etico interaziendale AOUC Città della Salute $\mathrm{e}$ della Scienza-A0 Ordine Mauriziano di Torino-ASL Città di Torino; De Medisch Ethisch Toetsingscommissie van Maastricht UMC. The study started enrolling patients in January 2021.

Trial registration number NCT04681534.
Strengths and limitations of this study

- New study protocols are necessary to ass outcomes form adaptive deep brain stimulation (aDBS) versus conventional DBS. This specific study assesses the safety and efficacy of aDBS using a new implantable device.

- The study includes patients with Parkinson's disease in the need of implantable pulse generators replacement, thus overcoming the limits of acute setting (stun effect) seen in de nove DBS patients.

- The use of an implantable device minimises risks for the patients, as compared with the previously used aDBS external devices.

- The number of patients is low but the results will help to design larger studies.

- This is the first study assessing the good on time with aDBS.

\section{INTRODUCTION}

Deep brain stimulation (DBS) is an established treatment for Parkinson's disease (PD), but its progress has been hampered by stagnation in methodological, technological and device development. DBS proved to be effective in improving major PD symptoms in long-term follow-up studies ${ }^{1-7}$ and currently, DBS is the surgical treatment of choice for patients with PD with medication-resistant motor fluctuations, dyskinesias and refractory tremor. ${ }^{1}$ In particular, DBS of the subthalamic nucleus (STN) has been shown to improve motor symptoms of PD, levodopa-induced complications and overall quality of life. ${ }^{7}$

However, current devices deliver conventional DBS (cDBS) with constant stimulation parameters, not adapting real-time to clinical features, but leaving to reprogramming 
visits the possibility to improve patient's response and satisfaction. $^{8}$

Limitations of cDBS include lack of responsiveness to patients' needs, fixed therapeutic window, repeated hospital visits for stimulation adjustment thus ultimately leading to suboptimal and more expensive therapy. ${ }^{8}$ In addition, the excessive and unnecessary electrical stimulation over time may interfere with the residual physiological functions of the basal ganglia, thus contributing ${ }^{9}$ to the development of neurological complications such as impairment of speech, balance and gait, and, possibly, cognition. In particular, the decline in verbal fluency, which is the most frequent side effect of STN-DBS, was associated with the influence of stimulation on sounding neural pathways. Some of these stimulation-related side effects can be reversed by reprogramming. ${ }^{10}$

A new approach to overcome cDBS limitations is now represented by adaptive DBS (aDBS) in which the intensity of stimulation is set automatically by real-time adaptation to the patient's clinical state, in a closed-loop fashion. ${ }^{11}{ }^{12}$ The patient's state is estimated by analysing the local neural activity (local field potentials, LFPs) recorded through the implanted DBS lead while stimulation is ON. ${ }^{13}$ Such biosignals, and more specifically the beta frequency band $(8-35 \mathrm{~Hz})$, are related to patient's clinical state and to levodopa intake,${ }^{14-16}$ and are involved in movement preparation and execution ${ }^{17-19}$ and more in general to motor state. ${ }^{20} 21$

LFPs-based aDBS has already been tested in humans, demonstrating to be effective in reducing motor symptoms of PD, comparable or even better than cDBS. ${ }^{20} 22-25$ In addition, it has been shown that aDBS significantly reduces side effects often associated with DBS therapy such as levodopa-induced dyskinesia ${ }^{25}$ and speech impairments. ${ }^{26}$

However, the information regarding the long-term safety and efficacy of aDBS remains limited. In fact, to date, studies comparing the efficacy and safety of aDBS to cDBS had intrinsic limitations, due to technical reasons. Initial studies were mostly performed in the immediate postoperative period, after surgery for DBS electrode implant, when the temporary presence of externalised electrodes allows the collection of data using external devices. This approach has several major limitations since symptom improvement may be in part attributed to lesional or implantation effects associated with surgery ${ }^{27} 28$ and the effects of DBS and adverse events (AEs) in the 'acute' (postoperative) period are known to differ from its 'chronic' effects. ${ }^{29}$ Recently, two studies confirmed the benefits of aDBS in patients at implantable pulse generator (IPG) replacement, ${ }^{30} 31$ and protocols studying aDBS in these patients have been proposed. ${ }^{32} \mathrm{In}$ addition, due to the lack of available implantable devices delivering aDBS, studies foresaw short periods of stimulation, with a maximum length of follow-up to 24 hours. ${ }^{30}$ Even though a new CE-marked implantable device able to record LFPs while DBS is ON (Medtronic Percept) has been recently introduced, no data on long-term aDBS is available as well as specific protocols to compare aDBS and cDBS.

Here, we present the protocol of a double-blind crossover study to assess the safety and potential benefits of aDBS delivered through a new implantable system capable of delivering both cDBS and aDBS, the AlphaDBS System (Newronika S.p.A.). This system will allow, for the first time, to overcome the limitations of the current experimental settings. Furthermore, in agreement with the results of basic research, we expect that the most interesting potential benefits of aDBS will be observed in the long-run, since aDBS may be able to improve axial signs and reduce fluctuations that are measured through patient's diaries and that cannot be assessed in the short term.

\section{Study objectives}

The aim of this study is to assess the safety and the potential efficacy of personalised LFP-based aDBS, using the implantable AlphaDBS System, in patients with PD, chronically implanted in the STN for DBS, at the time of IPG replacement.

The primary objective will be to evaluate the safety and tolerability of the AlphaDBS System, when used in cDBS and aDBS mode, based on the following endpoints:

- Occurrence of device-related AEs.

- Decrease in the total electrical energy delivered (TEED) to the patient.

As aDBS can be considered as a new treatment, all device related AEs will be reported and analysed against other devices on the market. Particular attention will be given to unexpected AEs and to those related to aDBS malfunctioning. In addition, TEED is an objective measure of the amount of energy transferred by DBS amplitude to the patient's brain. Previous works showed a significant reduction of TEED in aDBS compared with cDBS. Since TEED is correlated to dyskinesia occurrence, ${ }^{33}$ which is one of the stimulation-related side effects that aDBS may be able to control, ${ }^{25}$ TEED was also included as a quantitative safety endpoint.

Since this is the first study on the use of AlphaDBS System and on the chronic application of its aDBS implementation, secondary objective will be to evaluate the potential efficacy of aDBS and AlphaDBS System usability.

Efficacy will be evaluated from the following secondary measures:

- Evaluation of PD-related motor symptoms (ie, bradykinesia, rigidity and tremor at rest) and their fluctuations through repeated clinical assessments (using the Unified Parkinson's Disease Rating Scale MDSUPDRS-part III).

- Evaluation of dyskinesia and their fluctuations through repeated clinical assessments (using the Unified Dyskinesia Rating Scale (UDysRS) and wearable Systems).

- Evaluation of 'Time On' with and without dyskinesia and 'Time Off', assessed through Patient Diary. 


\begin{tabular}{|c|c|c|c|c|c|c|c|}
\hline Hospitalization & $\begin{array}{l}\text { Surgery: IPG } \\
\text { replacement }\end{array}$ & $\begin{array}{l}\text { Day } 1 \\
\text { Parameters } \\
\text { set up }\end{array}$ & $\begin{array}{c}\text { Experimental } \\
\text { session } 1 \\
\text { (cDBS OR } \\
\text { aDBS) }\end{array}$ & $\begin{array}{c}\text { Day } 3 \\
\text { Mode switch } \\
\text { Experimental } \\
\text { session 2 } \\
\text { (cDBS OR } \\
\text { aDBS) }\end{array}$ & \begin{tabular}{l}
\multicolumn{1}{|c|}{ Day 4} \\
Mode switch \\
Clinical \\
assessment \\
Discharge
\end{tabular} & $\begin{array}{c}\text { Day } 18 \\
\begin{array}{c}\text { Clinical } \\
\text { assessment }\end{array} \\
\begin{array}{c}\text { Mode } \\
\text { switch }\end{array} \\
\begin{array}{c}\text { Clinical } \\
\text { assessment }\end{array}\end{array}$ & $\begin{array}{c}\text { Clinical } \\
\text { assessment } \\
\text { Mode } \\
\text { switch }\end{array}$ \\
\hline
\end{tabular}

Figure 1 The trial time-line in patients participating to both the short-term and long-term follow-up phases: after completing the experimental procedures foreseen in day 1 , day 2 and day 3 , on day 4 , in the morning, the patient will be discharged with the AlphaDBSipg delivering aDBS or cDBS for 2 weeks. On day 18, the patient will undergo a clinical assessment. After the assessment the stimulation mode will be switched and the patient will undergo a new clinical assessment. If the second clinical assessment (with changed stimulation mode on) will be successfully completed, the patient will be discharged with the AlphaDBS device delivering aDBS or cDBS for additional 2 weeks. On day 32, the patient will undergo the last clinical assessment. aDBS, adaptive deep brain stimulation; cDBS, conventional DBS; IPG, implantable pulse generators.

Usability will be evaluated by means of usability questionnaires (see online supplemental material 1).

Exploratory objectives include evaluation of DBS associated deficits, through the DBS Impairment Scale (DBSIS) ${ }^{34}$ and evaluation of the effects of aDBS on speech.

Data collection using non-single patient use items, such as wearable systems and/or microphones that need to be sanitised, may be stopped in case of local COVID-19 emergency.

\section{Study design}

The study protocol is organised in two phases: the 'shortterm follow-up' and the 'long-term follow-up' (figure 1). During the 'short-term follow-up', fully eligible patients will be randomised to undergo a 2-day experimental sessions (ie, one per each type of stimulation mode, cDBS and aDBS), during hospitalisation, to collect information on safety and efficacy endpoints as assessed by experienced neurologists.

Patients who will not experience severe side effects during the 'short-term follow-up' and who will be deemed suitable by the neurologist, will be eligible to continue in the 'long-term follow-up' phase (1 month) in their 'home' environment. The AlphaDBS System will deliver the stimulation in aDBS or cDBS mode, for 2 weeks in each mode, following the same order as in the 'short-term follow-up'.

\section{METHODS AND PROCEDURE \\ Study centres}

The study involves six investigational sites (in Italy, Poland and The Netherlands). In particular, four centres are located in Italy (the University of Padua, the Fondazione IRCCS Ca' Granda Ospedale Maggiore Policlinico of Milan, the IRCCS Istituto Neurologico Besta of Milan, and the AOU Città della Salute e della Scienza of Torino), one in Poland (Narodowy Instytut Onkologii im. Marii Skłodowskiej-Curie, Warsaw) and one in The Netherlands (Maastricht UMC+, Maastricht).

\section{Inclusion criteria}

All patients included in the study must have been already implanted with DBS electrodes in the past. At the time of their first DBS implant (electrodes+first IPG now to be replaced), they were selected for DBS indication on the basis of the Core Assessment Programme for Surgical Interventional Therapies (CAPSIT) guidelines $\left(\right.$ CAPSIT-PD $\left.{ }^{35}\right)$. Even though some of the listed inclusion/exclusion criteria are similar to that used for DBS indication, we decided to reconsider them because of the time elapsed from DBS first implant.

- Diagnosis of idiopathic PD.

- Subject is bilaterally treated with DBS in the STN using a Medtronic Activa PC or Activa RC IPG (monochannel or dual channel).

- DBS implant for at least 3 years and in need of battery replacement within 12 months after consent;

- Patients must be able to understand and sign the informed consent document.

\section{Exclusion criteria}

- Patients with severe cognitive decline, as resulting from Montreal Cognitive Assessment (MoCA) assessment (MoCA score $<10)$.

- Patients with major psychiatric issues or any other condition that, based on the physician opinion, could interfere with the study conduct (eg, severe depression, psychosis).

- Patients with any medical conditions potentially interfering with DBS battery replacement surgery (eg, severe hypertension, active cancer, intake of drugs interfering with the coagulation).

- Need to replace or reposition the leads during the IPG replacement procedure.

- Patients with $>10$ recurrent falls experienced in the 3 months prior to consent.

- Patients that cannot tolerate an interruption of DBS stimulation for at least $30 \mathrm{~min}$. 


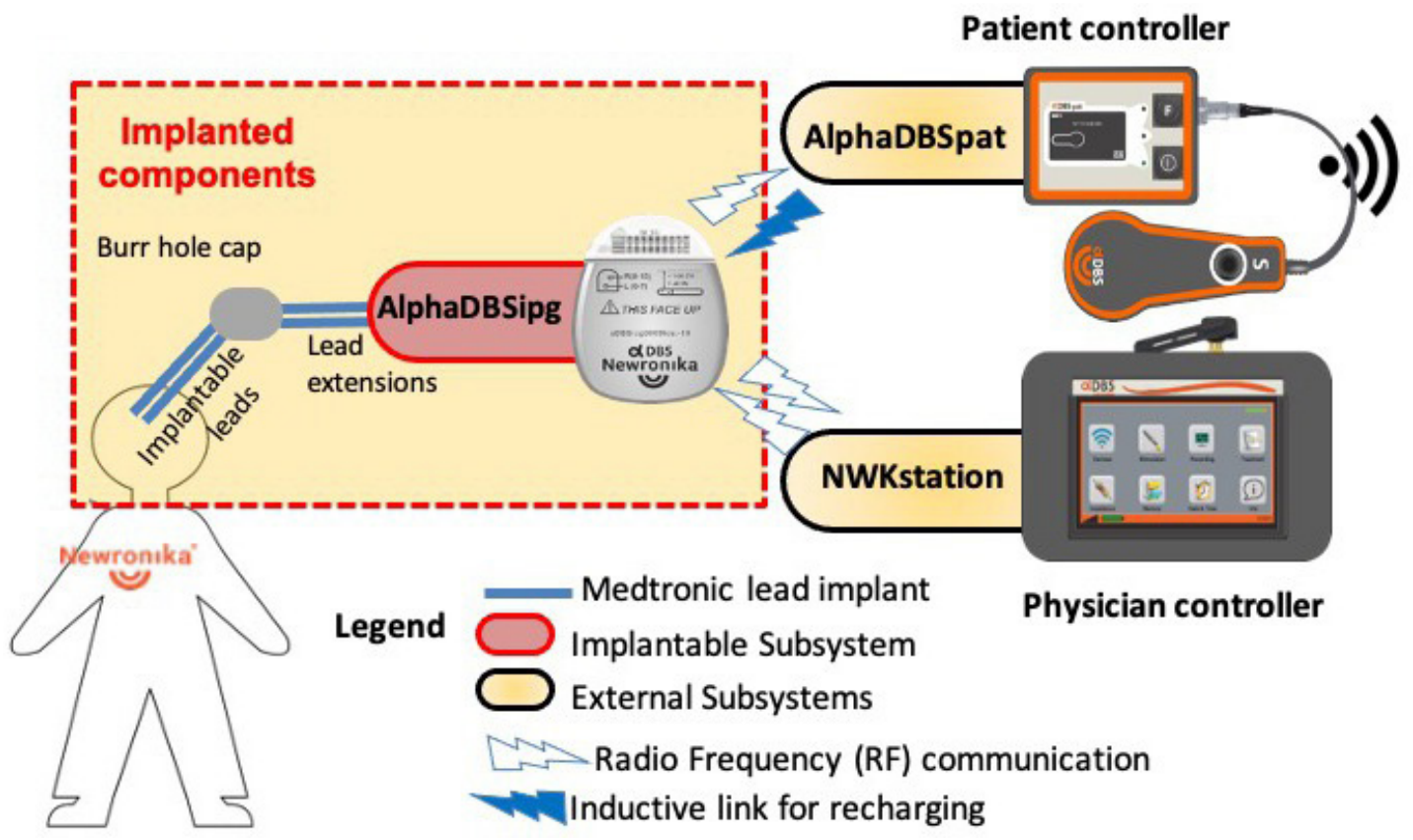

Figure 2 AlphaDBS system overview. AlphaDBS, alpha deep brain stimulation; RF, radiofrequency.

- Patients taking less than one levodopa dose per day.

- Patients with no LFPs recorded intraoperatively from any contacts pair, during the IPG replacement procedure.

- Pregnant or breastfeeding women.

According to exclusion criterion in the second bullet point, the neurologist has the possibility to exclude patients whenever a condition reducing the compliance is observed, including moderate cognitive impairment.

\section{Device description}

The AlphaDBS System is a DBS system that includes the possibility for the neurologist to programme the stimulation in conventional mode (cDBS) or in adaptive, closedloop, mode (aDBS). When the AlphaDBS System is used in aDBS mode, it delivers DBS stimulation using an intelligent biofeedback mechanism to automatically modulate stimulation. AlphaDBS is able to record and analyse in real-time LFPs while DBS in ON from the same implanted lead, and automatically adjust stimulation.

The AlphaDBS System is composed of different subsystems (figure 2): the AlphaDBSipg (IPG delivering stimulation in aDBS or cDBS mode and recording/analysing LFPs from implanted DBS leads); AlphaDBSpat (external patient controller); NWKstation (external physician controller).

The AlphaDBSipg is an active implantable medical device that applies cDBS/aDBS. It is powered by a hermetically sealed rechargeable battery within a titanium case. The AlphaDBS System, manufactured by Newronika SpA (Milan, Italy), is CE-marked.

In cDBS mode, the AlphaDBSipg, with 16 independent stimulation current controlled outputs, delivers asymmetric biphasic balanced constant current pulse train. Stimulation can be delivered in bipolar or monopolar configuration by selecting a contact pair or one contact in each of the two available leads (stimulation parameters: pulse width (us), amplitude (V) and frequency (Hz)). In monopolar stimulation, the reference electrode is simulated by the IPG enclosure.

In aDBS mode, an adaptive algorithm will use LFP signals from implanted electrodes extracting information to decrease the energy of stimulation (amplitude) when the patient is responding appropriately to pharmacological therapy and increasing the energy when the patient's symptoms are not well controlled. The algorithm that will be used in aDBS mode will be personalised based on LFP modulation in the $13-35 \mathrm{~Hz}$ frequency band (beta band), as described elsewhere. ${ }^{36}$

The AlphaDBS System has several innovative features that implement a distributed architecture allowing data collection and management that make it a reliable platform for aDBS and closed-loop neuromodulation applications. Major innovations reside in the technology for artifact-free recordings $^{36-38}$ that is stimulation agnostic, electrode configuration independent and needless for back-end processing. This implies that LFPs can be recorded with stimulation $\mathrm{ON}$ from all contact pairs, not necessarily symmetrical around the stimulation contact, and with different stimulation types. In addition, the artefact rejection methodology is implemented at the chip level and not at the system level, thus leaving the whole computational capacity free for closed-loop algorithm implementation. Another important feature is the ability of the system both to provide on-demand real-time streaming of LFPs both in ON and OFF without the need of additional receivers worn by the patient, but using directly the clinician controller (NWKStation) and to provide continuous embedded data storage that is always 
ON 24/7 whatever the stimulation mode (OFF, cDBS, aDBS). Thanks to the data management infrastructure, the embedded data storage guarantees no data loss for memory overwriting because data are automatically downloaded to the patient controller (AlphaDBSpat) during recharging, using the same device. This is crucial to allow full biomarker tracking for future aDBS optimisation.

\section{Evaluations and procedures}

After providing consent, each patient will undergo a Screening Period, during which demographic information and additional information on the medical management will be collected. Each patient will undergo a series of screening evaluations, including: evaluation of battery level, medical history, physical, neurological and psychiatric examinations to assess cognitive decline (ie, MoCA) and major psychiatric issues (eg, severe depression, psychosis), as suggested in CAPSIT-PD guidelines, ${ }^{35}$ measurement of vital signs (as performed in normal clinical practice before IPG replacement surgery), assessment of prior and concomitant medications, of AEs occurring after giving informed consent, and evaluation of MDSUPDRS and UDysRS at (1) stim-ON/med-OFF, (2) stim-OFF (1 hour)/med-OFF, (3) stim-OFF/med-ON, (4) stim-ON (1 hour)/med-ON. The med-ON condition will be evaluated after the administration of a LEED morning dose $+30 \%$.

Patients with a confirmed need for battery replacement will be qualified for surgery. Hospitalisation will be conducted in agreement with local standard practice for IPG replacement.

On day 0 , during routine surgery for IPG replacement, after IPG removal, the exposed leads will be connected to temporary extensions in order to check the integrity of the leads and the occurrence of ECG artefacts. The patients with ECG artefacts impairing LFP recording will not be excluded and will receive a standard of care new IPG implant. Otherwise, the patient will be enrolled.

The day after surgery (day 1), the patients will undergo personalised algorithm setup. LFPs will be recorded synchronously, through the AlphaDBSipg device, for about $30 \mathrm{~min}$, from all available electrode pairs in the med-OFF/stim-OFF condition (no DBS and no levodopa) to establish (1) the best recording pair, (2) the peak LFPs frequency and (3) the LFPs band of interest. Then, a routine DBS current titration session will be performed to establish both the optimal cDBS parameters with AlphaDBSipg, and the therapeutic window. Finally, the AlphaDBS System will be calibrated using the personalised beta band and peak previously defined.

At the end of the personalised algorithm setup, patients will be assigned to cDBS; randomisation to aDBS or cDBS treatment will take place on the following day.

On two consecutive days after the algorithm setup (day 2 and day 3) aDBS and cDBS will be tested, one stimulation mode per day, according to the randomisation schedule.

The experimental session will start around 7:30 hour (expected time) and will last for about 9 hours (figure 3 ). At the end of the experimental session, the stimulation will continue overnight until the next washout period in the same mode.

At the beginning of the session, the stimulation will be switched off for at least $30 \mathrm{~min}$ of stimulation washout (stim-OFF/med-OFF condition), and then switched on.

Each experimental session will include the following assessments:

- T0: before the administration of the morning dopaminergic therapy and after at least $30 \mathrm{~min}$ of stimulation washout (stim-OFF/med-OFF)-UDysRS, MDS-UPDRS III and AEs recording, speech analysis.

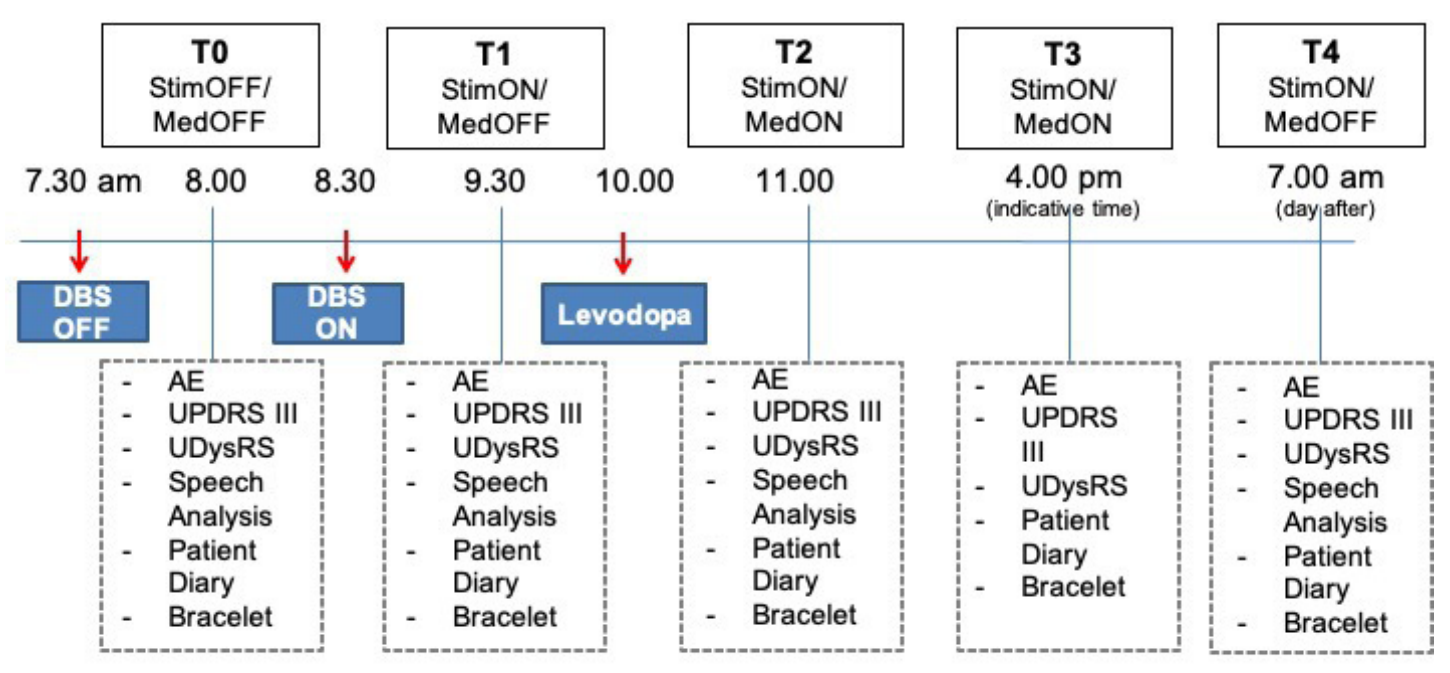

Time estimated for each visit: about 30 minutes if includes «speech analysis», otherwise 15 minutes.

Figure 3 Summary of examinations foreseen at each time point of the experimental sessions (day 2 and day 3 ). Note that timing is indicative and may vary up to 45 min per session. AE, adverse event; DBS, deep brain stimulation; UDysRS, Unified Dyskinesia Rating Scale; UPDRS, Unified Parkinson's Disease Rating Scale. 
- T1: before the administration of the morning dopaminergic therapy and after 1 hour of active stimulation (stim-ON/med-OFF)—UDysRS, MDS-UPDRS III and $\mathrm{AE}$ recording, speech analysis.

- T2: around 1 hour after dopaminergic therapy administration, when the effect of dopaminergic therapy will reach its best effect (stim-ON/med-ON)—UDysRS, MDS-UPDRS III, AE recording, speech analysis.

- T3: in the afternoon, around 16:00 hour or if the patient therapeutic schedule foresees a second dopaminergic therapy, when the effect of the therapy will reach its best effect (stim-ON/med-ON)-UDysRS, MDS-UPDRS III and AE recording.

- T4: the following day (day 3 or day 4), in the morning, before starting any experimental procedure, when the stimulation is still $\mathrm{ON}$, and before the administration of the morning dopaminergic therapy (stim-ON/medOFF)—UDysRS, MDS-UPDRS III and AEs recording, speech analysis.

The timing of the assessments is indicative and variations up to $45 \mathrm{~min}$ are allowed.

Throughout the experimental session, to monitor motor symptoms fluctuations, the patient will wear a bracelet equipped with a three-axial accelerometer and will fill in his/her patient's diary, for the whole duration of the experimental session. Speech analysis will be performed with Semantic and phonemic evaluations will be recorded with the Verbal Fluency (VF) test (DelisKaplan Executive Function System), and control word repetition tasks.

The parameters to calculate the TEED at T4 will be automatically collected from the AlphaDBS System.

On day 4, if the neurologist will deem the patient suitable for the 'long-term follow-up' phase, the patient will undergo another clinical assessment and will be discharged. The clinical assessment will take place about 1 hour after mo rning dopaminergic therapy administration, when the effect of the therapy will reach its best effect (stim-ON/med-ON), administering UDysRS and MDS-UPDRS III scales and examining possible side effects. After the visit, the stimulation will be automatically switched to the stimulation mode, randomly allocated on day 2.

After the examination, patients (blinded to treatment) will receive the training on how to use the device, and then be discharged from the hospital and sent to their 'home' environment for 2 weeks in each stimulation mode.

During this follow-up period, a research fellow/nurse will monitor the patient remotely every day to assess the patient status, check Concomitant Medications and record AEs.

After 2 weeks of treatment, on day 18, the patient will provide diaries completed in the last 3 days before the visit and will undergo a clinical assessment (performed by a blinded neurologist) in stim ON-med ON including UDysRS, MDS-UPDRS III and DBS-IS scales, collection of possible side effects, speech analysis and TEED (automatically collected from the AlphaDBSipg). Also, the patient/caregiver will provide his/her inputs related to the System usability for what concerns the IPG recharging process.

After the visit, the stimulation will be automatically switched to the other stimulation mode (as in day 3), and the patient will be sent home. The same protocol will be followed for 2 weeks until day 32 . Then, the patient will be able to choose whether to keep the AlphaDBS System or replace it with a compatible commercially available IPG.

\section{Randomisation}

Each recruited patient will be randomly assigned to one of the stimulation modes to be allocated as a first treatment, based on a centre-specific computer-generated randomisation list.

Each eligible patient will be recorded on the online electronic case report form (eCRF) system and a progressive study number will be automatically assigned. If the patient is eligible, the investigators will randomise him/ her and the eCRF will display a randomisation code corresponding to the first free number from the randomisation list.

At the beginning of each experimental day, the designated person in charge of DBS programming

(unblind), will use the randomisation code as PIN code to enter the Physician Programmer (NWKStation), to programme the system in cDBS or aDBS according to randomisation.

\section{Methods: statistical methods and data management Sample size}

The objective of the study is to collect data, such as the degree of correlation between GOT in aDBS and cDBS, that will allow calculation of the sample size needed for a pivotal study if this study confirms the results obtained in a previous trial. This study will randomise at least 15 patients.

Based on the figures obtained in the clinical trial with patients in the 'acute' phase, ${ }^{25}$ and without considering corrections for multiple testing, this sample will allow using exploratory statistics to demonstrate a difference in TEED during cDBS and aDBS sessions through a non-parametric test for an effect size of 1.14, assuming the following parameters, using type I error probability equal to 0.05 and power of $99 \%$ : TEED $=44.6$ in aDBS, TEED $=158.7$ in cDBS, $\mathrm{SD}=100$, multiplying by 4.5 the higher SD observed. Also, 15 patients will allow to observe AEs occurring in 5\%-10\% of the patients, but not rare events in the range $1 \%-2 \%$. However, at this stage, rare hardware-related AEs (1\%-2\%) are not considered since they were already described by other DBS devices manufacturers and thus expected. Also, rare hardware related are usually observable in studies with longer observation periods than that included in this study (1 month) which is thus not ideal to assess this type of information, which in any case will be collected. 
Data collection and management

All study data will be collected and stored through online eCRFs. The system will provide a safe environment suitable for multicentre studies, with deidentified patients' data and clinical forms for data collection that can be shared among different operative units, allowing CRF signature and modifications tracking. A CRO is in charge of data management and quality assurance.

\section{Monitoring}

The study monitoring will be conducted in agreement with Good Clinical Practice regulations (ISO 14155:2011). The designated CRO will oversee the conduct of the trial. The Study Monitor will maintain contact with the Investigator and will visit the study site for the purpose of discussing and/or retrieving data. An initiation (prestudy) visit will be made by the study monitor to discuss with the Investigator the protocol and the obligations of both the Sponsor and the Investigator. The study monitor will perform periodic, interim monitoring visits. In case that on-site monitoring visits cannot be completed, Remote Monitoring Visit will be implemented and conducted according to the Standard Operating Procedure of the CRO in charge of study monitoring (eg, during sanitary emergency).

\section{Data analysis}

The CRO will carry out all steps of analysis related to clinical efficacy and safety assessment.

The effect of randomisation will be explored by descriptive statistics analysing the clinical endpoints (ie, UDysRS, MDS-UPDRS III, etc) in Stim-OFF/Med-OFF condition before aDBS and cDBS experimental sessions.

Safety will be evaluated on all patients randomised and receiving at least one of the treatments. It will include the comparison of: (1) TEED delivered to the patient during aDBS and cDBS experimental sessions, (2) AEs during the two treatments.

This is a first in man study not designed to claim efficacy of aDBS or superiority of aDBS over cDBS. Exploratory analysis will be only performed in order to obtain summary data to inform decisions on future clinical development phases. Clinical efficacy will be evaluated through intention-to-treat analysis.

Differences in clinical endpoints when patients receive aDBS or cDBS will be compared, as well as the time courses of MDS-UPDRS III scores, motor symptoms fluctuations, 'Time Off'/'Time On' and UDysRS during aDBS and cDBS treatments. Data will be compared with repeated measures general linear model analyses. Tukey's honest significance test will be used for post hoc analysis. Differences will be considered significant at $\mathrm{p}<0.05$ for the generation of hypotheses.

Since the protocol is a first in man study for the AlphaDBSipg, it includes various and repeated assessments to better evaluate patient's tolerability and response. However, these can be burdensome for the patients and minor protocol deviations might be expected. Minor deviations will be included in the analysis whereas major deviations will be excluded.

\section{Ethics and dissemination}

Risk-benefit analysis

Potential risks and benefits of aDBS will be clearly explained to the patients in the Informed Consent Form that will be provided at screening, prior to start the study protocol.

If the results of the trial will be promising, patients with PD will have a new innovative device for DBS that will allow the delivery of aDBS. In any case, new long-term LFP recordings will be available thanks to the implantation of the AlphaDBS system thus improving the understanding of PD neurophysiology.

Patients treated with aDBS could experience a reduction of symptoms, better quality of life, and a simplification of patient management, reducing the number of visits and calls to the treating neurologist to fine-tune DBS programming settings. In addition, patients involved in the study could experience personal benefits, possibly including: overall reduction of the electrical energy delivered to the tissues, and of the patient's OFF time (compared with cDBS), overall increase of the patient's ON time without troublesome dyskinesia, improvement of efficacy in reducing bradykinesia, rigidity, and tremor (compared with cDBS), reduction 'levodopa-induced dyskinesia', improvement in speech, balance and gait problems related to stimulation.

Given the extensive bench testing and animal and clinical studies conducted, there is a reasonable expectation that the device will be technically successful and that it will function as intended.

The replacement of a DBS IPG involves risks, and we expect that the patient implanted with the AlphaDBSipg will be exposed to the same procedure-related risks reported for other DBS Systems on the market. These risks are the ones commonly associated with IPG replacement surgery. An additional risk may occur in patients choosing to replace the AlphaDBSipg with a commercial IPG at the end of the long-term follow-up.

COVID-19 seriously impacted on the conduction of experimental trials and research activity. ${ }^{39}$ A COVID-19 risk assessment, related to the study conduct was prepared, in agreement with the indications provided in the 'Guidance on the management of clinical trials during the COVID-19 (coronavirus) pandemic (V.3, 28 April 2020)' issued by the European Commission and coordinated by EMA.

\section{Informed consent, IEC/IRB approval and MoH approval}

The study will be carried out in accordance with the Declaration of Helsinki, as amended by the 64th General Assembly of the World Medical Association, Fortaleza, Brazil, October 2013.

The protocol, Subject Information Sheet, Informed Consent Form and the Data Privacy Consent Form were reviewed and approved, prior to initiating any trial-related 
activity, by the Ethical Committees of each institution involved namely: Comitato Etico Milano Area 2 (Milano), Comitato Etico Fondazione IRCCS Istituto Neurologico C. Besta (Milano), Comitato Etico Interaziendale A.O.U. Città della Salute e della Scienza di Torino-A.O. Ordine Mauriziano-A.S.L. Città di Torino (Torino), Comitato Etico per la Sperimentazione Clinica della Provincia di Padova (Padova); Bioethics Committee at the National Institute of Oncology of Maria Skłodowska-Curie (Warsaw), De Medisch Ethisch Toetsingscommissie van Maastricht UMC (The Netherlands). As the AlphaDBS System is an investigational device, the trial required the approval, as premarket study, of competent authorities, namely: the Italian Ministry of Health, Directorate General for Medical Devices and Pharmaceutical service, the Polish Office for Registration of Medicinal Products, Medical Devices and Biocidal Products and the Dutch Central Committee on Research Involving Human Subjects.

\section{Patient and public involvement}

Patients from an Italian PD association provided inputs on the definition of relevant benefits related to the results of this aDBS investigation and on device usability.

\section{Author affiliations}

${ }^{1}$ Dipartimento di Ingegneria e Architettura, Università degli Studi di Trieste, Trieste, Italy

${ }^{2}$ UO Neurofisiopatologia, Fondazione IRCCS Ca' Granda Ospedale Maggiore Policlinico, Milan, Italy

${ }^{3}$ Newronika SpA, Milan, Italy

${ }^{4}$ Fundación del Hospital Nacional de Parapléjicos para la Investigación y la Integración, Toledo, Spain

${ }^{5}$ CINAC, Hospital Universitario HM Puerta del Sur, Universidad CEU-San Pablo, Móstoles, Madrid, Spain

${ }^{6}$ Division of Neurosurgery, Department of Surgery, Toronto Western Hospital, University of Toronto, Toronto, Ontario, Canada

${ }^{7}$ Krembil Research Institute, University Health Network, Toronto, Ontario, Canada

${ }^{8}$ Grenoble Institute of Neurosciences, INSERM U1216, University Grenoble Alpes, Grenoble, France

${ }^{9}$ Department of Neurology, University of Wurzburg, Würzburg, Germany

${ }^{10}$ ASST Santi Paolo e Carlo, Milano, Italy

${ }^{11}$ Aldo Ravelli Research Center for Neurotechnology and Experimental

Neurotherapeutics, Department of Health Sciences, University of Milan, Milan, Italy

Contributors SM and CC ideated and designed the protocol, wrote the protocol and documentation for regulatory purposes and ethical committee approvals, and drafted the manuscript. OS, MA, LR and AP ideated and designed the protocol. AML, GF, EM and JV critically reviewed the protocol procedures and manuscript. All authors reviewed and approved the final version of this manuscript.

Funding This study is sponsored by Newronika SpA, a spin off company of the Fondazione IRCCS Ca' Granda Ospedale Maggiore Policlinico of Milan and of the University of Milan. Award/Grant number is not applicable.

Disclaimer All the scientific findings derived from this protocol are aimed to be made public through publication of articles in international journals.

Competing interests All the scientific findings derived from this protocol are aimed to be made public through publication of articles in international journals. Conflict of Interest statement: AP, GF and SM are founders and shareholders of Newronika SpA, and are member of Newronika's scientific advisory board. LR is founder, shareholder and CEO of Newronika SpA. MA and OS are stock option holder and work for Newronika S.p.A. CC works for Newronika SpA. EM is member of the scientific advisory board of Newronika SpA, JV is member of the scientific advisory board of Newronika SpA and works as a consultant to Boston Scientific and Medtronic, and has received honoraria for lectures from Boston Scientific and Medtronic as well as research grants from Boston Scientific and Medtronic, AML is member of the scientific advisory board of Newronika SpA, has served as a consultant for Boston Scientific, Medtronic, Aleva, and Abbott and is a cofounder of Functional Neuromodulation.

Patient consent for publication Not applicable.

Provenance and peer review Not commissioned; externally peer reviewed.

Supplemental material This content has been supplied by the author(s). It has not been vetted by BMJ Publishing Group Limited (BMJ) and may not have been peer-reviewed. Any opinions or recommendations discussed are solely those of the author(s) and are not endorsed by BMJ. BMJ disclaims all liability and responsibility arising from any reliance placed on the content. Where the content includes any translated material, BMJ does not warrant the accuracy and reliability of the translations (including but not limited to local regulations, clinical guidelines, terminology, drug names and drug dosages), and is not responsible for any error and/or omissions arising from translation and adaptation or otherwise.

Open access This is an open access article distributed in accordance with the Creative Commons Attribution Non Commercial (CC BY-NC 4.0) license, which permits others to distribute, remix, adapt, build upon this work non-commercially, and license their derivative works on different terms, provided the original work is properly cited, appropriate credit is given, any changes made indicated, and the use is non-commercial. See: http://creativecommons.org/licenses/by-nc/4.0/.

ORCID iD

Sara Marceglia http://orcid.org/0000-0002-0456-866X

\section{REFERENCES}

1 Krack P, Martinez-Fernandez R, del Alamo M. Current applications and limitations of surgical treatments for movement disorders: surgical treatments for movement disorders. Mov Disord 2017;32:36-52.

2 Kleiner-Fisman G, Herzog J, Fisman DN, et al. Subthalamic nucleus deep brain stimulation: summary and meta-analysis of outcomes. Mov Disord 2006;21 Suppl 14:S290-304.

3 Bronstein JM, Tagliati M, Alterman RL, et al. Deep brain stimulation for Parkinson disease: an expert consensus and review of key issues. Arch Neurol 2011;68:165.

4 Deuschl G, Schade-Brittinger C, Krack P, et al. A randomized trial of deep-brain stimulation for Parkinson's disease. $N$ Engl J Med 2006;355:896-908.

5 Rodriguez-Oroz MC, Moro E, Krack P. Long-term outcomes of surgical therapies for Parkinson's disease. Mov Disord 2012;27:1718-28.

6 Odekerken VJJ, Boel JA, Schmand BA, et al. Gpi vs STN deep brain stimulation for Parkinson disease: three-year follow-up. Neurology 2016;86:755-61.

7 Cavallieri F, Fraix V, Bove F, et al. Predictors of long-term outcome of subthalamic stimulation in Parkinson disease. Ann Neurol 2021;89:587-97.

8 Koeglsperger T, Palleis C, Hell F, et al. Deep brain stimulation programming for movement disorders: current concepts and evidence-based strategies. Front Neurol 2019;10:410.

9 Chen CC, Brücke C, Kempf F, et al. Deep brain stimulation of the subthalamic nucleus: a two-edged sword. Curr Biol 2006;16:R952-3.

10 Picillo M, Lozano AM, Kou N, et al. Programming deep brain stimulation for Parkinson's disease: the Toronto Western Hospital algorithms. Brain Stimul 2016;9:425-37.

11 Priori A, Foffani G, Rossi L, et al. Adaptive deep brain stimulation (aDBS) controlled by local field potential oscillations. Exp Neurol 2013;245:77-86.

12 Mitchell KT, Starr PA. Smart neuromodulation in movement disorders. In: Handbook of clinical neurology. Elsevier, 2020: 153-61.

13 Marceglia S, Rossi L, Foffani G, et al. Basal ganglia local field potentials: applications in the development of new deep brain stimulation devices for movement disorders. Expert Rev Med Devices 2007;4:605-14.

14 Arlotti M, Rosa M, Marceglia S, et al. The adaptive deep brain stimulation challenge. Parkinsonism Relat Disord 2016;28:12-17.

15 Brown P. Oscillatory nature of human basal ganglia activity: relationship to the pathophysiology of Parkinson's disease. Mov Disord 2003;18:357-63.

16 Priori A, Foffani G, Pesenti A, et al. Rhythm-specific pharmacological modulation of subthalamic activity in Parkinson's disease. Exp Neurol 2004;189:369-79.

17 Marceglia S, Fumagalli M, Priori A. What neurophysiological recordings tell us about cognitive and behavioral functions of the human subthalamic nucleus. Expert Rev Neurother 2011;11:139-49. 
18 Marceglia S, Fiorio M, Foffani G, et al. Modulation of beta oscillations in the subthalamic area during action observation in Parkinson's disease. Neuroscience 2009;161:1027-36

19 Foffani G, Bianchi AM, Baselli G, et al. Movement-Related frequency modulation of beta oscillatory activity in the human subthalamic nucleus. J Physiol 2005;568:699-711.

20 Arlotti M, Marceglia S, Foffani G, et al. Eight-hours adaptive deep brain stimulation in patients with Parkinson disease. Neurology 2018;90:e971-6.

21 Neumann W-J, Degen K, Schneider G-H. Subthalamic synchronized oscillatory activity correlates with motor impairment in patients with Parkinson's disease: Correlation of Subthalamic B Oscillations and PD Symptoms. Mov Disord 2016;31:1748-51.

22 Little S, Pogosyan A, Neal S, et al. Adaptive deep brain stimulation in advanced Parkinson disease. Ann Neurol 2013;74:449-57.

23 Little S, Beudel M, Zrinzo L, et al. Bilateral adaptive deep brain stimulation is effective in Parkinson's disease. J Neurol Neurosurg Psychiatry 2016;87:717-21.

24 Rosa M, Arlotti M, Ardolino G, et al. Adaptive deep brain stimulation in a freely moving parkinsonian patient. Mov Disord 2015;30:1003-5.

25 Rosa M, Arlotti M, Marceglia S, et al. Adaptive deep brain stimulation controls levodopa-induced side effects in parkinsonian patients. Mov Disord 2017;32:628-9.

26 Little S, Tripoliti E, Beudel M, et al. Adaptive deep brain stimulation for Parkinson's disease demonstrates reduced speech side effects compared to conventional stimulation in the acute setting. J Neurol Neurosurg Psychiatry 2016;87:1388-9.

27 Mann JM, Foote KD, Garvan CW, et al. Brain penetration effects of microelectrodes and DBS leads in STN or GPI. J Neurol Neurosurg Psychiatry 2009;80:794-8.

28 Mestre TA, Lang AE, Okun MS. Factors influencing the outcome of deep brain stimulation: placebo, nocebo, lessebo, and lesion effects. Mov Disord 2016;31:290-8.

29 Rosa M, Giannicola G, Servello D, et al. Subthalamic loca field beta oscillations during ongoing deep brain stimulation in
Parkinson's disease in hyperacute and chronic phases. Neurosignals 2011;19:151-62.

30 Arlotti M, Palmisano C, Minafra B, et al. Monitoring subthalamic oscillations for 24 hours in a freely moving Parkinson's disease patient. Mov Disord 2019;34:757-9.

31 Piña-Fuentes D, Little S, Oterdoom M, et al. Adaptive DBS in a Parkinson's patient with chronically implanted DBS: a proof of principle. Mov Disord 2017;32:1253-4.

32 Piña-Fuentes D, Beudel M, Little S, et al. Adaptive deep brain stimulation as advanced Parkinson's disease treatment (adapt study): protocol for a pseudo-randomised clinical study. BMJ Open 2019;9:e029652.

33 Prenassi M, Arlotti M, Borellini L, et al. The relationship between electrical energy delivered by deep brain stimulation and levodopainduced dyskinesias in Parkinson's disease: a retrospective preliminary analysis. Front Neurol 2021;12:643841.

34 Maier F, Lewis CJ, Eggers C, et al. Development and validation of the deep brain stimulation impairment scale (DBS-IS). Parkinsonism Relat Disord 2017;36:69-75

35 Defer GL, Widner H, Marié RM, et al. Core assessment program for surgical interventional therapies in Parkinson's disease (CAPSIT-PD). Mov Disord 1999;14:572-84.

36 Arlotti M, Rossi L, Rosa M, et al. An external portable device for adaptive deep brain stimulation (aDBS) clinical research in advanced Parkinson's disease. Med Eng Phys 2016;38:498-505.

37 Priori A, Foffani G, Rossi L. Apparatus for treating neurological disorders by means of adaptive electro-stimulation retroacted by biopotentials, 2005. Available: https://patents.google.com/patent/ US20080269836A1/en

38 Rossi L, Foffani G, Marceglia S, et al. An electronic device for artefact suppression in human local field potential recordings during deep brain stimulation. J Neural Eng 2007;4:96-106.

39 Priori A, Baisi A, Banderali G, et al. The many faces of Covid-19 at a glance: a university hospital multidisciplinary account from Milan, Italy. Front Public Health 2021;8:575029. 